\title{
Multiplexed FBG sensor recorded in multimode microstructured polymer optical fibre
}

\author{
Ian P. Johnson ${ }^{\mathrm{a}}$, David J. Webb ${ }^{\mathrm{a}}$, Kyriacos Kalli ${ }^{\mathrm{b}}$, Maryanne C. J. Large ${ }^{\mathrm{c}}$, Alexander Argyros ${ }^{\mathrm{c}}$ \\ ${ }^{a}$ Photonics Research Group Aston University, Aston Triangle Birmingham, B4 7ET, UK \\ ${ }^{\mathrm{b}}$ Nanophotonics Research Laboratory, Cyprus University of Technology, Cyprus \\ ${ }^{\mathrm{c}}$ Institute of Photonics and Optical Science, University of Sydney, NSW 2006 Australia
}

\begin{abstract}
Fibre Bragg gratings have been inscribed in multimode microstructured polymer optical fibre (POF), with a core size of $50 \mu \mathrm{m}$. The microstructured POF (mPOF) consists of a three ring hole structure and is made purely from poly(methyl methacrylate) (PMMA). In comparison to silica fibre, POF has a much smaller Young's modulus and a much greater breaking strain; additionally multimode fibre holds advantages of ease of handling and launching conditions. A linear strain sensitivity of $1.32 \pm 0.01 \mathrm{pm} / \mu \varepsilon$ has been measured in the range 0 to $2 \%$ strain.
\end{abstract}

The fibre drawing process leads to a degree of molecular alignment along the fibre axis. This alignment can be thermally annealed out; this can induce a permanent blue shift in the Bragg wavelength of a grating fabricated prior to annealing by up to $20 \mathrm{~nm}$. Utilising this, wavelength demultiplexed gratings can be fabricated using a single phase mask. As an illustration of this we present for the first time wavelength division multiplexing of the spectral response of three Bragg gratings in POF within the C-band region.

Complementing this work, a technique of splicing mPOF to step index silica fibre is described using UV curing optical adhesive, allowing characterisation of Bragg gratings fabricated in this fibre.

Keywords: POF, mPOF, PCF, WDM, FBG, large strain sensing, polymer splice, thermal annealing

\section{INTRODUCTION}

To date grating sensor research activity has mainly concentrated on silica fibre Bragg grating (FBG) sensors. In recent years however this mature technology in silica fibre has started to be transferred to polymer optical fibre (POF), taking advantage of the polymer material properties. A FBG sensor was first fabricated in step index POF in $1991^{[1]}$ and was later followed by the fabrication of the first FBG sensors in single mode microstructured polymer optical fibre (mPOF) ${ }^{[2]}$. The majority of POF is based upon poly(methyl methacrylate) (PMMA), which holds a number of advantages over its silica counterpart. Polymers are clinically acceptable and furthermore are flexible and non-brittle making them highly advantageous for in-vivo sensing applications ${ }^{[3]}$. Polymer has a Young's modulus which is 25 times smaller than that of silica ${ }^{[4],[5]}$. This has enabled demonstrations of grating tuning by recoverable strains of up to $13 \%$ by Lui et al ${ }^{[6]}$. Furthermore Bragg gratings fabricated in POF have demonstrated a negative temperature coefficient of $-43 \mathrm{pm} /{ }^{\circ} \mathrm{C}^{[7]}$ compared to silica fibre which is approximately $10 \mathrm{pm} /{ }^{\circ} \mathrm{C}$.

Fibre Bragg gratings fabricated in $\mathrm{mPOF}$ and particularly multimode $(\mathrm{MM}) \mathrm{mPOF}$ are of particular interest as they have the potential to offer low cost sensing. Microstructured POF compared to silica fibre is easier to fabricate and the chemical composition of PMMA offers the possibility of chemical modification using organic techniques. As the mPOF is multimode the task of connecting to silica couplers, light sources and optical spectrum analysers is made easier. The MMmPOF gives possibilities of using ferrule connectors ${ }^{[8]}$, where slight misalignment in connecting fibres would be insignificant compared to the impracticalities of using single mode fibre this way. Using multimode POF also lends itself nicely to cheaper light sources, especially when comparing the costs of a broad area emitter to a single transverse mode light source.

In this paper it is reported for the first time the fabrication of wavelength division multiplexed (WDM) FBG sensors in MMmPOF. The ability of tuning a Bragg grating within a $20 \mathrm{~nm}$ range is discussed as a technique of fabricating a

Photonic Crystal Fibers IV, edited by Kyriacos Kalli, Waclaw Urbanczyk, Proc. of SPIE Vol. 7714, 77140D · C 2010 SPIE · CCC code: 0277-786X/10/\$18 · doi: 10.1117/12.854410 
WDM sensor using a single phase mask during the UV inscription. We also report on the strain sensitivity of Bragg gratings fabricated in MMmPOF and the relaxation of the fibre after large strains of up to 5\% have been applied. Complementing this working is the first account of splicing polymer optical fibre to silica fibre using UV curing optical adhesive. This will allow Bragg gratings fabricated in MMmPOF to be characterised in applications outside of the laboratory.

\section{UV INSCRIPTION}

Fibre Bragg gratings were fabricated in pure PMMA MMmPOF. The fibre has an outer diameter of $150 \mu \mathrm{m}$ and a core diameter of $50 \mu \mathrm{m}$, the core being surrounded by three rings of holes as shown in Figure 1. The high refractive index core is contained by the lower effective refractive index of the ring of holes, providing index guiding in the core. The fibre manufacturing is described by Barton et al without the sleeving procedure ${ }^{[3]}$.

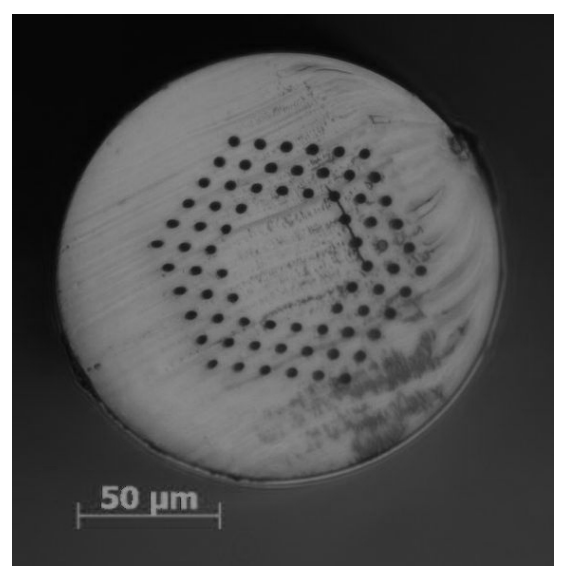

Figure 1 Microscope image of end face of MMmPOF

A helium-cadmium ( $\mathrm{HeCd}$ ) laser with an ultraviolet (UV) output of $30 \mathrm{~mW}$ at a wavelength of $325 \mathrm{~nm}$ is used to inscribe Bragg gratings in the MMmPOF. A stationary beam is focused vertically downwards using a cylindrical lens with a $10 \mathrm{~cm}$ focal length, exposing the fibre through a phase mask with a period of $1057.2 \mathrm{~nm}$. The grating length is defined by the width of the UV beam, which in this case is $1.8 \mathrm{~mm}$. Contradictory to common silica Bragg grating inscription, the MMmPOF is laid on a v-groove and taped down using polyimide tape. This is a relatively simple UV inscription set-up; however the essence of the set-up is the stability. Inscription times using this type of fibre are on average one hour and during this time the POF needs to be immobile and any drooping prevented to ensure alignment of the UV beam into the core of the POF throughout the inscription. Alignment of the UV beam to the core of the MMmPOF is achieved by monitoring the reflection scattering from the fibre. With previous experience aligning to the core using this method can be highly successful, with a success rate of approximately 95\%. Using this MMmPOF is a secure option to inscribe Bragg gratings in POF due to the robustness of the fibre and also the large core diameter, this fibre is often used within our laboratory when examining the UV laser inscription set-up.

\section{BRAGG GRATINGS IN MMmPOF}

The reflected spectrum and growth curve of a Bragg grating inscribed in MMmPOF for 55 minutes is shown in Figure $2 \mathrm{a}$ and Figure $2 \mathrm{~b}$ respectively. The Bragg wavelength of gratings inscribed using the described method and phase mask are in the region of $1562 \mathrm{~nm}$. Typically the reflection spectrum has a signal-to-noise ratio of $20 \mathrm{dBm}$ and a FWHM of $4 \mathrm{nmwhen}$ using a bandwidth of $0.5 \mathrm{~nm}$ on the OSA, the light source has a typical spectrum density of $11 \mathrm{dBm} / \mathrm{nm}$ between $1540-1600 \mathrm{~nm}$. There is a large bandwidth compared to what is commonly seen in a single mode response as a larger number of modes are being reflected in the MMmPOF. For this reason the Bragg wavelength is calculated by taking the average between the data points on the rising and falling edges of the reflection spectrum $3 \mathrm{~dB}$ below the peak value. Significant growth can be seen around 25 minutes, with a sharp increase in strength 
between 35 and 55 minutes. There is a jump in the growth around 32 minutes as the interrogation setup was adjusted to optimise launching conditions.
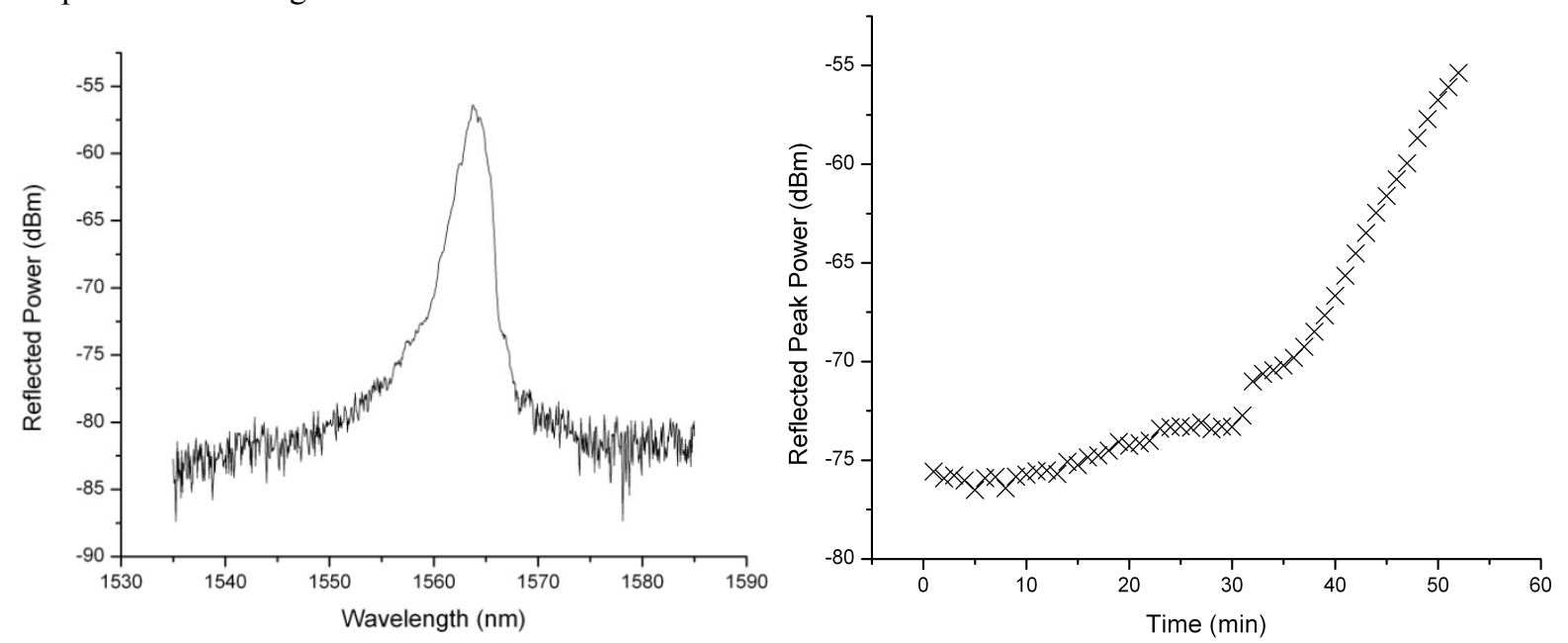

Figure 2 a: reflection spectrum of Bragg grating fabricated in MMmPOF. b: Growth curve of the reflection signal during 55 minutes of exposure.

Figure $2 \mathrm{a}$ and Figure $2 \mathrm{~b}$ were obtained by interrogating the Bragg grating in reflection both during and after inscription. This was accomplished using a MM 50:50 silica coupler, a broadband light source (BBS) and an optical spectrum analyzer (OSA). The BBS (Thorlabs ASE-FL7002-C4) is first launched into a 20 metre spool of MM $50 / 125 \mu \mathrm{m}$ silica fibre to ensure even modal distribution. The OSA (HP 70004A) has MM input capability to ensure the true modal distribution of the reflected spectrum is captured. A connection is made between the arm of the silica coupler and the POF using a FC/APC connector on the silica fibre which was then butt coupled to the POF. A small amount of polymer index matching gel was used in the coupling to curtail Fresnel reflections. High-quality end faces of the POF are essential for effective butt-coupling; these were prepared using an $80^{\circ} \mathrm{C}$ hot cleave as described by Law et $a l^{[11]}$; Figure 1 shows the end face of the MMmPOF prepared using this technique.

\section{UV ADHESIVE SPLICING OF MMmPOF TO MM SILICA OPTICAL FIBRE}

To realise the full potential of FBGs in MMmPOF the technology needs to progress out of the laboratory and into the real world. This has been achieved by developing a technique of splicing POF to step index silica fibre using a UV curing optical adhesive. The optical adhesive used is Loctite 3525; Table 1 shows the main qualities of this adhesive.

Table 1 Summary specification of Loctite $3525^{[12]}$

\begin{tabular}{|lr|c|}
\hline Refractive Index of uncured material & 1.48 \\
\hline Viscosity & $@ 25^{\circ} \mathrm{C}, \mathrm{mPa} \cdot \mathrm{s}(\mathrm{cP})$ & $9,500-21,000$ \\
\hline Refractive Index of cured material & $\mathrm{N} / \mathrm{mm}^{2}$ & 1.51 \\
\hline Tensile Strength, at break, ISO 527-3 & $\mathrm{psi}$ & 24 \\
\cline { 3 - 3 } & $\mathrm{N} / \mathrm{mm}^{2}$ & 1750 \\
\cline { 3 - 3 } & $\mathrm{psi}$ & 25,000 \\
\hline Tensile Modulus, ISO 527-3 & $43^{\circ} \mathrm{C}$ \\
\hline Glass Transition Temperature & \multicolumn{2}{|l}{} \\
\hline
\end{tabular}

As with butt-coupling, the key to a good splice is high-quality end faces of both the polymer and silica fibres. Again both end faces of the POF are prepared using the $80^{\circ} \mathrm{C}$ hot cleave method ${ }^{[11]}$. However now a FC/APC connector is not used on the silica fibre, in its place bare silica fibre is used with an $8^{\circ}$ cleave to reduce the Fresnel reflections from the silica end face. The cleaver (HSAFC-2008) is provided by Oxford Fiber. The polymer and silica fibres are placed on separate v-grooves and aligned on a precision translation stage. Alignment is first achieved launching red 
light into the MMmPOF from the silica fibre using a red LED fault finder light source and monitoring the guidance of the red light within the core of the MMmPOF by projecting the end face onto a screen using a $\times 20$ microscope objective. Once the end face of the polymer and silica fibre are aligned and brought close up to each other so no visible gap can be seen, a small amount of Loctite 3525 adhesive is applied to the polymer-silica joint. Coupling is now optimised by making minor adjustments in the fibre alignment to maximise the Bragg reflection spectrum when interrogating the inscribed grating, using the 50:50 MM silica coupler as described previously. Once the alignment is optimised the Loctite 3525 is UV cured. The curing is accomplished using an Omni Cure Series 1000, UV curing light source, exposing the glued joint for 10 minutes. The source had a $2 \%$ iris coverage which provided a measured optical output power of $60 \mathrm{~mW}$.

Initial development of the splicing technique proved troublesome. In preliminary work, bending the MMmPOF resulted in significant change in modal distribution visible in the reflected spectrum resulting in variations of $300 \mathrm{pm}$ in the recovered wavelength. The reasons for this may possibly be because of the travel of the adhesive along the ring of holes of the MMmPOF. As can be seen in Figure 3a, in this example the adhesive has travelled $180 \mu \mathrm{m}$ along the length of the MMmPOF. This results in an interruption in the guidance at the glued joint, therefore as the fibre is bent there is modal redistribution and a loss of light at the joint. Hence as is shown in Figure $3 \mathrm{~b}$ there is a change in the reflection spectrum as the MMmPOF is bent in various positions.
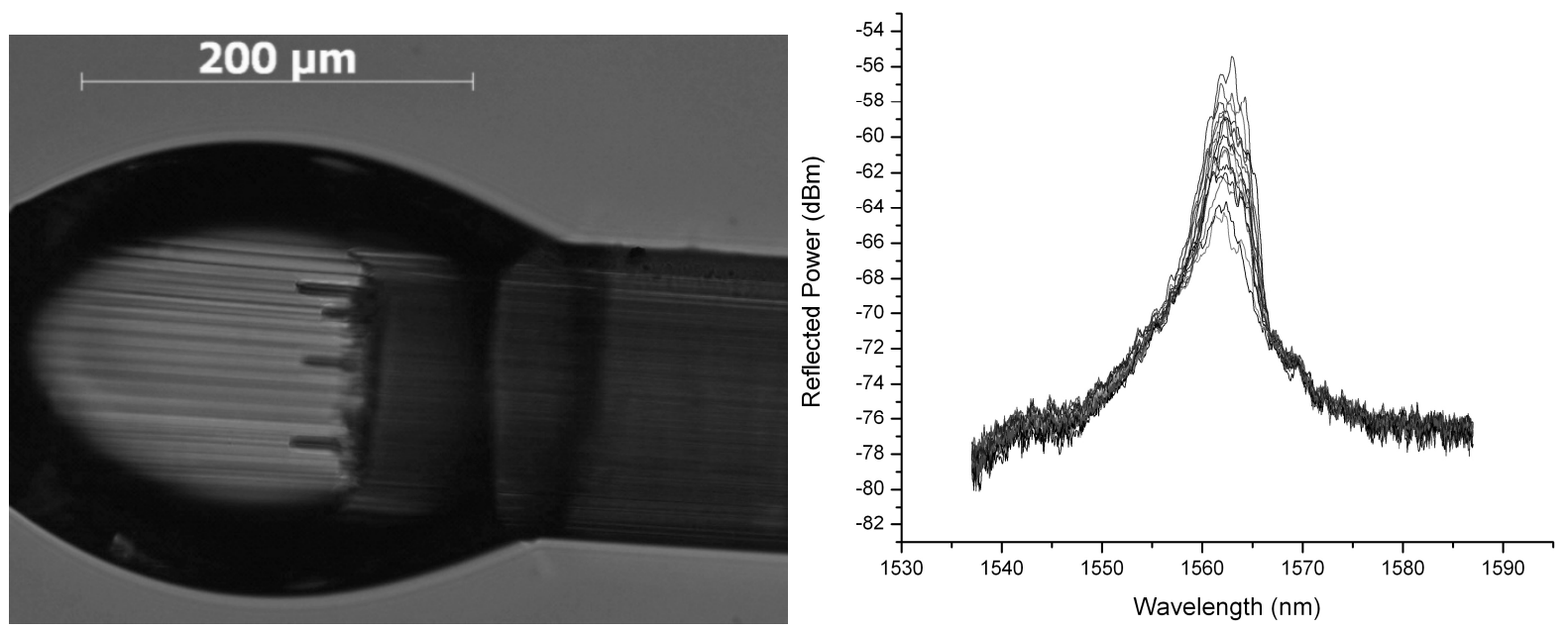

Figure 3 a: Loctite 3525 UV curing adhesive (light colour) has travelled along the holes of mPOF b: resolution of $300 \mathrm{pm}$ caused by changes in spectral profile due to interruption of guidance at glued joint

Initially the UV curing adhesive was applied to the end face of the cleaved silica fibre before aligning to the MMmPOF; however this allowed a substantial amount of time for the adhesive to flow along the holes of the mPOF. The UV adhesive splicing was optimised by first aligning the silica fibre to the MMmPOF with no adhesive applied, after which a tiny amount of adhesive is applied while interrogating the Bragg grating reflection. This small amount of adhesive is then quickly UV cured and the polymer and silica fibres are fixed together in a much shorter amount of time. A second coat of adhesive is then applied to the joint; here a larger amount of adhesive is used to support the joint. Figure 4 compares the reflected Bragg spectra before and after the UV adhesive is finally cured after an initial 1 minute cure to grip the two fibres and a second supporting cure of ten minutes. As can be seen there is an increase in the reflected power of the spectrum once the UV adhesive has been cured, this is believed to be due to the refractive index of the adhesive increasing from 1.48 to 1.51 when cured, as stated in Table 1. Nevertheless there appears to be minimal loss due to the adhesive with any losses being generated from the modal mismatching at the glued joint. Additionally the resolution using the optimised adhesive splicing is significantly better with no change in the reflected spectrum due to bending of the MMmPOF, giving a resolution an order of magnitude smaller than previously measured. Figure $4 \mathrm{~b}$ shows MMmPOF that has been UV spliced to silica optical fibre, with the entire polymer fibre and glue joint suspended in air. 

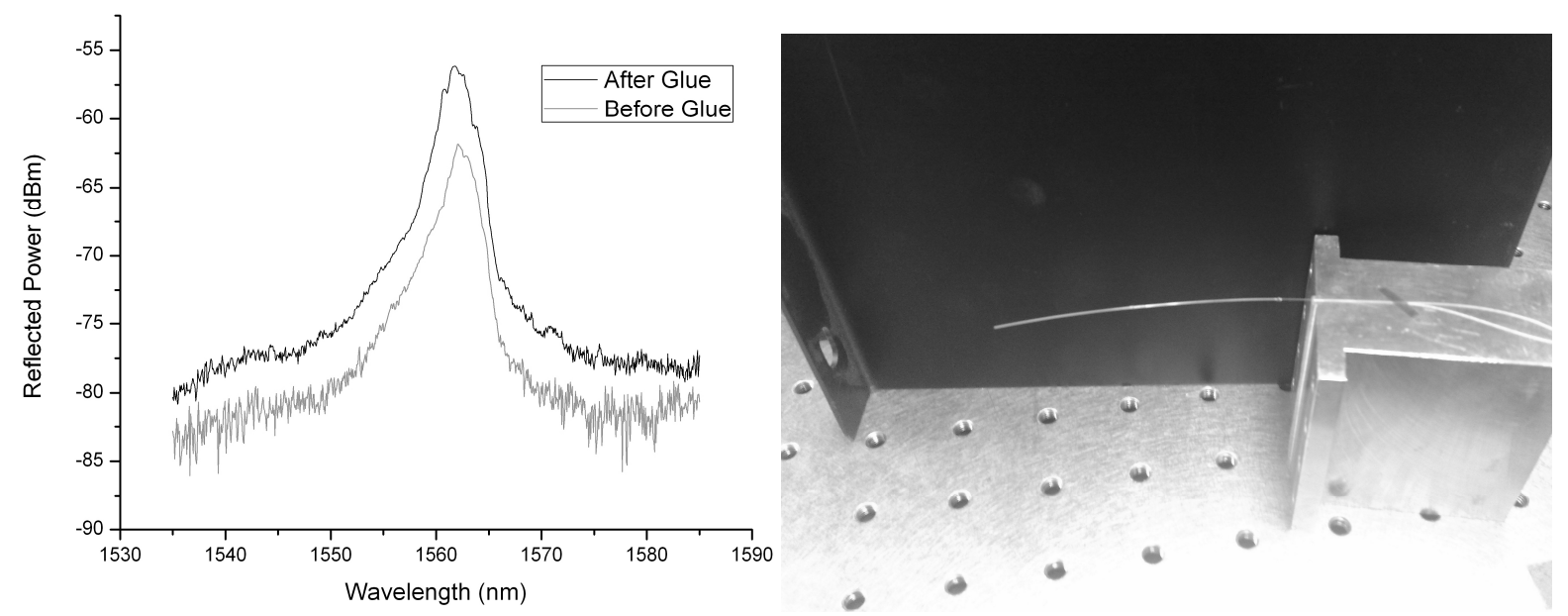

Figure 4 a: Comparison of reflected Bragg spectra before and after UV adhesive splicing b: photograph of MMmPOF UV adhesive spliced to silica fibre.

\section{WAVELENGTH DIVISION MULTIPLEXED FBG SENSOR}

As was investigated by Carroll et al ${ }^{[13]}$ a permanent blue wavelength shift can be induced in the reflection spectrum of a Bragg grating in polymer fibre by utilising the thermal properties of POF. Carroll discussed that during manufacture, polymer optical fibre is pulled under tension. This tension tends to align the molecular chains along the axis of the fibre. When the POF is heated a temperature will be reached where the molecule chains will start to relax from their orientation along the fibre axis, causing the fibre to permanently shrink. It is believed the temperature at which shrinkage of the POF will appear is dependent on the thermal history of the polymer and the drawing specifications, as previously shown by Ishigure $e a l^{[14]}$ and additionally by UV exposure during the Bragg grating inscription by Shafee ${ }^{[15]}$.

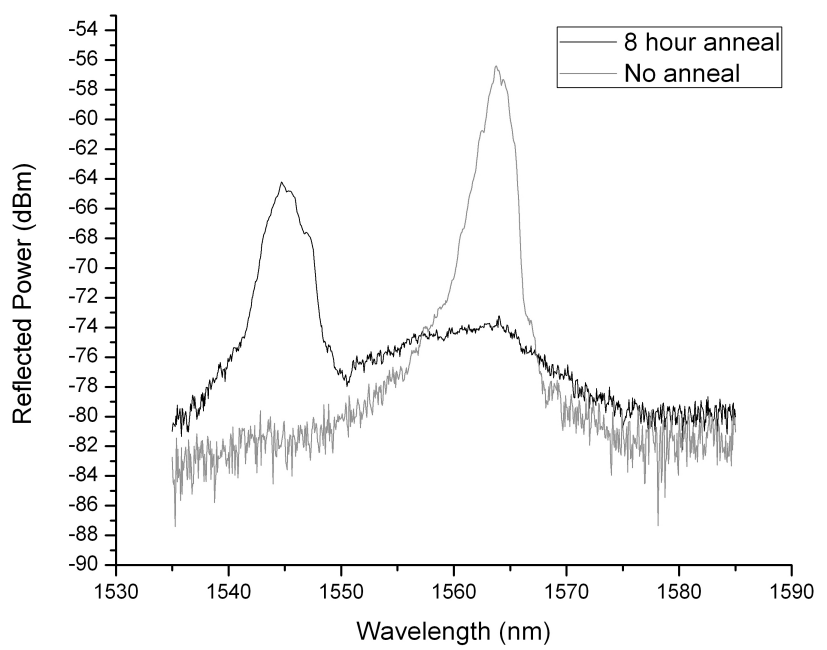

Figure 5 The effect of thermal annealing a Bragg grating in MMmPOF for 8 hours.

The effect of thermally annealing a length of MMmPOF at a constant temperature of $80^{\circ} \mathrm{C}$ for eight hours can be seen in Figure 5. Firstly a Bragg grating is UV inscribed into the MMmPOF which is shown with the grey spectrum, this was inscribed using a phase mask with period $1057.2 \mathrm{~nm}$ producing a Bragg wavelength of $1562 \mathrm{~nm}$. The black spectrum shows the response from the same grating after thermal annealing; here the Bragg wavelength is $1545 \mathrm{~nm}$. 
Therefore thermally annealing the MMmPOF at $80^{\circ} \mathrm{C}$ for 8 hours has induced a permanent blue wavelength shift of $18 \mathrm{~nm}$. As discussed by Carroll ${ }^{[13]}$, it is believed the permanent wavelength shift is induced by the permanent shrinkage of the MMmPOF, which results in a decrease of the UV inscribed grating period $(\Lambda)$.

Thermally annealing the MMmPOF now gives the opportunity to tune the reflected Bragg wavelength of an UV inscribed Bragg grating. This feature has been utilised to fabricate the first wavelength division multiplexed (WDM) FBG sensor in MMmPOF using a single phase mask. Figure 6a shows the previously seen Bragg reflection with a $\lambda_{B}$ of $1545 \mathrm{~nm}$, which has been permanently shifted from $1562 \mathrm{~nm}$ when UV inscribed with a phase mask $(\Lambda=1057.2 \mathrm{~nm})$. The same phase mask is then used to UV inscribe a second Bragg grating in the same MMmPOF with a $\lambda_{\mathrm{B}}$ of $1562 \mathrm{~nm}$. As can be seen in Figure $6 \mathrm{~b}$ a WDM Bragg sensor has been inscribed in the MMmPOF using the single phase mask $(\Lambda=1057.2 \mathrm{~nm})$. Finally a third Bragg grating is UV inscribed into the same length of MMmPOF using a second phase mask with a shorter period of $\Lambda=1034.2 \mathrm{~nm}$. This generates a third Bragg response with a $\lambda_{\mathrm{B}}$ of $1528 \mathrm{~nm}$, as can be seen in Figure $6 \mathrm{c}$. Therefore three Bragg gratings have been UV inscribed into a single length of MMmPOF creating a WDM Bragg sensor. All three gratings were UV inscribed as previously described, with each Bragg grating taking approximately one hour to achieve the maximum signal-to-noise ratio before saturation. The three gratings each have a separation of ten millimetres, which was achieved by moving the MMmPOF along the v-groove in between individual inscriptions.
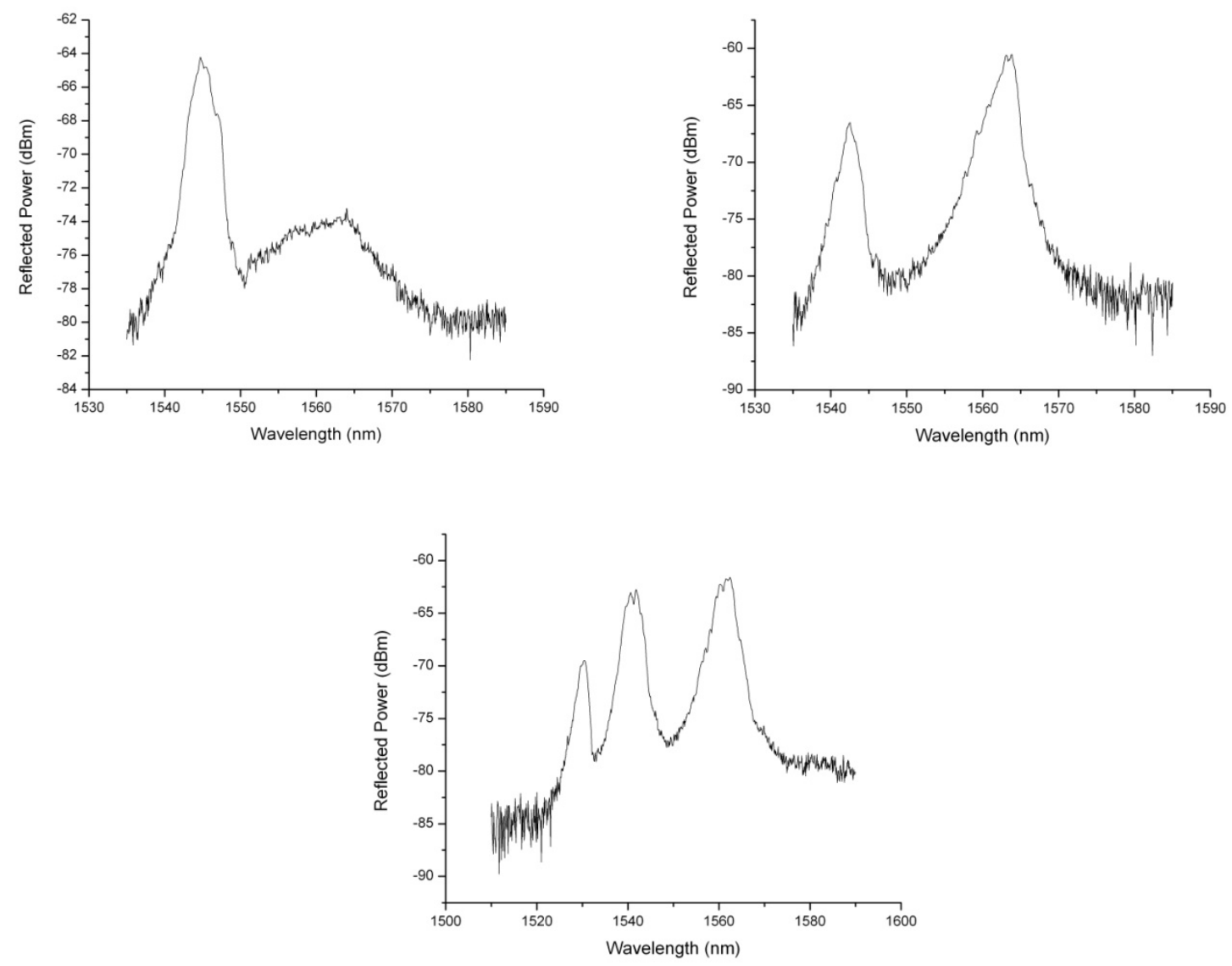

Figure 6 - a: A single Bragg grating thermally annealed to induce an $18 \mathrm{~nm}$ blue wavelength shift b: a second Bragg grating is inscribed into the same MMmPOF using the same phase mask c: a third Bragg grating is inscribed in the fibre using a second phase mask. 


\section{STRAIN CHARACTERISTICS OF A BRAGG GRATING SENSOR}

Included in this work are strain data from a single Bragg grating which has been UV inscribed in MMmPOF. Figure 7 shows the reflection spectrum of a Bragg grating fabricated using a phase mask $(\Lambda=1034.2 \mathrm{~nm})$ and the standard UV inscription previously described with an inscription time of around one hour. The Bragg response has a Bragg wavelength $\left(\lambda_{B}\right)$ of $1529 \mathrm{~nm}$, a FWHM of $3 \mathrm{~nm}$ and a signal-to-noise ratio of $15 \mathrm{dBm}$. Once inscribed the FBG was UV adhesive spliced to a MM 50/125 $\mu \mathrm{m}$ silica pigtail using the technique described earlier. The sensor was then glued to a translation stage for strain sensitivity characterisation using Araldite adhesive. Additional Araldite was applied around the glue joint between the polymer and silica fibre to support the splice further, preventing any movement of either fibre in close proximity of the joint.

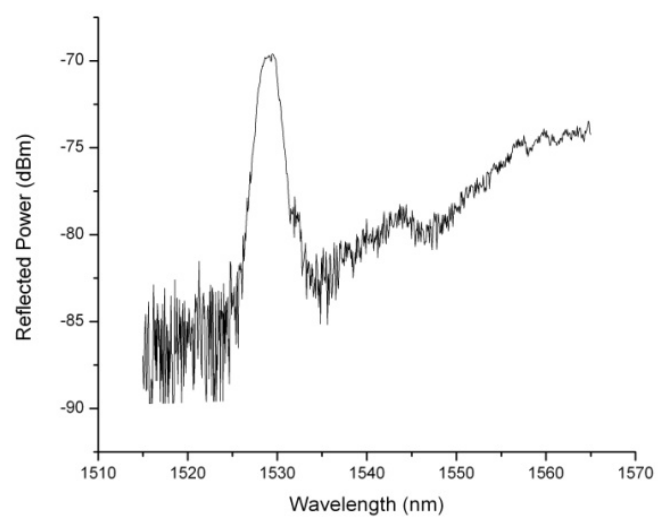

Figure 7 - Reflection spectrum of a Bragg grating UV inscribed in MMmPOF used for strain sensitivity characterisation.

The distance between the two stages, where the MMmPOF was suspended was $42 \mathrm{~mm}$. The MMmPOF was strained to $2 \%$ of this distance, which is $0.84 \mathrm{~mm}$. The strain is incremented in 14 steps of $0.06 \mathrm{~mm}$, one minute is allowed for incrementing the strain and capturing the reflected spectrum on a PC. The strain is then decreased in 14 steps of $0.06 \mathrm{~mm}$ and one minute is allowed between decreasing the strain and capturing the reflection spectrum on a PC. The strain was both incremented and decremented by hand using the micrometer of the translation stage. The Bragg wavelength $\left(\lambda_{B}\right)$ for each spectrum at each strained state is calculated using the $-3 \mathrm{~dB}$ average as described earlier. Figure 8 illustrates the change in $\lambda_{B}$ due to the increase and decrease in strain. Increasing the length of the suspended MMmPOF by $2 \%$, the equivalent of applying 20 milli-strain $(\mathrm{m} \varepsilon)$ induces a red wavelength shift of $26 \mathrm{~nm}$. The calculated strain sensitivity of the incremented strain is $1.3 \mathrm{pm} / \mu \varepsilon$. When decreasing the strain the MMmPOF is able to return to its original length in a relatively short amount of time. However there is a small amount of hysteresis when returning to $0 \%$ from $0.14 \%$, it is believed this is due the viscoelastic properties of PMMA and if the MMmPOF had been allowed to relax for a further five minutes the Bragg wavelength at $0 \%$ strain would have returned to its original value. 


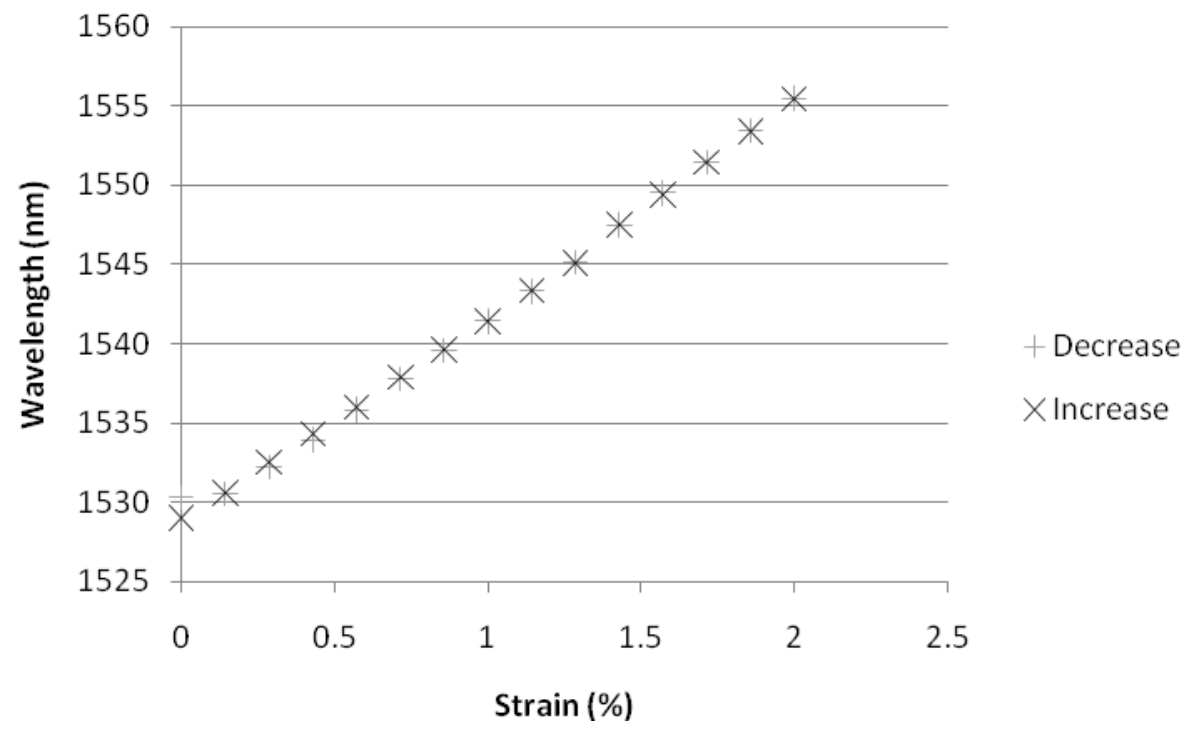

Figure 8 - Maximum strain of 2\% applied to MMmPOF FBG sensor

Figure 9 illustrates the situation where the suspended length of MMmPOF is strained to $5 \%$ of its length, which is $2.1 \mathrm{~mm}$. The strain was incremented in 30 steps of $0.07 \mathrm{~mm}$ and once more one minute was allowed between applying the strain and capturing the reflection spectrum on a PC. There is no shift in the Bragg wavelength when increasing from 0 to $0.167 \%$ as the fibre was slack when no strain was applied due to previous experiments straining the MMmPOF. The slack appears to be taken up when $1.67 \mathrm{~m} \varepsilon$ is applied. Thereafter there is a calculated strain sensitivity of $1.58 \mathrm{pm} / \mu \varepsilon$, inducing a red wavelength shift of $72 \mathrm{~nm}$ when extending the suspended fibre length by $4.8 \%$. When decreasing the strain, the strain is decremented in 30 steps of $0.07 \mathrm{~mm}$, again one minute is allowed between decreasing the strain and capturing the reflection spectrum of the Bragg grating on a PC. As can be seen in Figure 1 there is large hysteresis when decreasing the strain at this rate. Because the MMmPOF is a viscoelastic material and a large amount of strain has been applied to the polymer a considerable amount of time is needed to allow the MMmPOF to relax. When the strain is relaxed to $1.4 \%$ the MMmPOF becomes slack as can be seen with the change in the Bragg wavelength response. Here after there is only a small change in the Bragg wavelength due to the MMmPOF relaxing. It is expected to fully allow the MMmPOF to relax and recover to the original state 48 hours might be needed. 


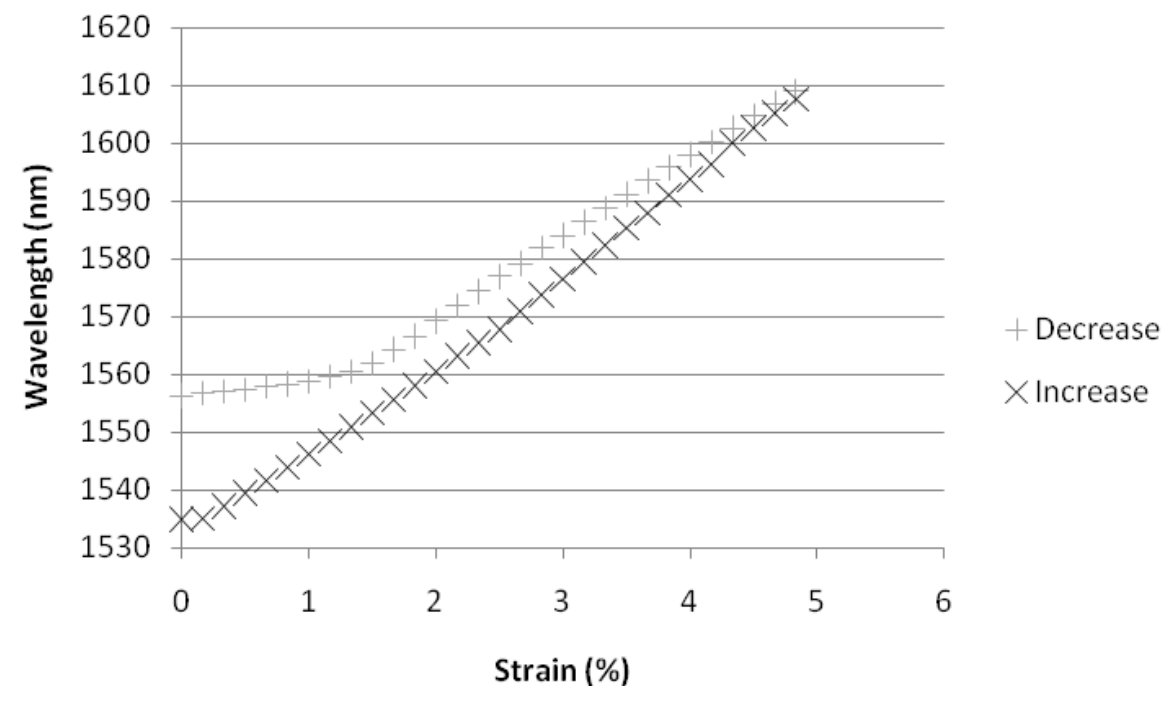

Figure 9 - Maximum strain of 5\% applied to MMmPOF FBG sensor

\section{Conclusions}

It has been demonstrated that Bragg gratings can be consistently fabricated in MMmPOF, due to its ease of handling, simple alignment during UV inscription and ease of launch conditions. Bragg gratings can be fabricated with a $20 \mathrm{dBm}$ signal-to-noise ratio with a FWHM of $4 \mathrm{~nm}$ at a Bragg wavelength of $1562 \mathrm{~nm}$ following on average a 60 minute inscription time. It has been seen that thermally annealing a pre-fabricated Bragg grating in MMmPOF will induce a permanent blue Bragg wavelength shift of $18 \mathrm{~nm}$. Utilising the thermal annealing properties, wavelength demultiplexed gratings have been fabricated using a single phase mask. Furthermore for the first time wavelength division multiplexing of the spectral response of three Bragg gratings fabricated in MMmPOF within the C-Band spectral region has been demonstrated. The linear strain sensitivity for a single Bragg grating was also calculated to be $1.3 \mathrm{pm} / \mu \varepsilon$ when applying between 0 to $2 \%$ strain. When characterising the strain sensitivity in the range of 0 to $5 \%$ the calculated sensitivity is $1.58 \mathrm{pm} / \mu \varepsilon$. Hysteresis can be observed when decreasing the strain from 5 to $0 \%$ as the fibre was not allowed enough time to relax after the relatively high strain with the fibre becoming slack when the strain was decreased to $1.4 \%$. This hysteresis might be eliminated if the fibre was given time to relax. Time is needed for the fibre to relax because of its viscoelastic characteristics. It is estimated that for the fibre to recover from an applied linear strain of 5\%, 48 hours would be needed.

In addition, an effective method of splicing polymer optical fibre to silica fibre has been developed using UV curing optical adhesive. Negligible loss due to the glued joint was observed, with any losses experienced due to modal mismatching. This gluing technique has enabled FBGs fabricated in MMmPOF and other polymer optical fibres to move out of the laboratory and into real applications, realising the full potential of polymer optical fibre. 


\section{ACKNOWLEDGEMENTS}

The authors would like to acknowledge the European Commission for the funding for this work via the Framework 7 project PHOtonic Skins For Optical Sensing (PHOSFOS). Additionally IPJ would like to acknowledge the UK Engineering and Physical Sciences Research Council (EPSRC), for student funding.

\section{REFERENCES}

[1] Z. Xiong, G.D. Peng, B. Wu, P.L. Chu, "Highly tunable Bragg gratings in single-mode polymer optical fibers" IEEE Photon. Technol. Lett. 11, 352-354 (1999).

[2] H. Dobb, D.J. Webb, K. Kalli, A. Argyros, M.C.J. Large, M.A. van Eijkelenborg "Continous wave ultraviolet light-induced fiber Bragg gratings in few and single mode microstructured polymer optical fibers" Opt. Lett. 30, 3296-3298 (2005).

[3] F. Baldini \& A.G. Mignani, 'Optical-Fiber Medical Sensors.' Materials Research Society, Volume 27, Number 5, 383-387. May 2002.

[4] M. Siliva-Lopez, A. Fender, W. MacPherson "Strain and temperature sensitivity of a single-mode polymer optical fiber" Opt Lett. 30(23), 3129-3131 (2005).

[5] G.W. Kaye, T.H. Laby [Tables of physical and chemical constants] Longmann, 45 (1995).

[6] H.L. Liu, H.Y. Liu, G.D. Peng, P.L. Chu, "Strain and temperature sensor using a combination of polymer and silica fibre Bragg gratings" Opt. Commun, 219, 139-142 (2003).

[7] Harbach NG. Fiber Bragg Gratings in Polymer Optical Fibers. Thèse EPFL, no 4021 (2008).

[8] R. Lwin, A. Argyros "Connecting Microstructured Polymer Optical Fibres to the World|" Proc. POF2009 Poster 7 Australia (2009)

[9] H.B. Liu, G.D. Peng, P.L. Chu, “Thermal tuning of polymer optical fiber Bragg gratings" IEEE Photon. Technol Lett, 13, 824(2001).

[10] G. Barton, M.A. van Eijkelenborg, G. Henry, M.C.J. Large, J. Zagari, "Fabrication of microstructured polymer optical fibers" Opt. Fiber Technol. 10, 325-335 (2004).

[11] S.H. Law, J.D. Harvey, R.J. Kruhlak, E.W.M.Song, G.W. Barton, M.A. van Eijkelenborg, M.C.J. Large, "Cleaving of microstructured polymer optical fibres" Opt. Commun. 258, 193-202 (2206).

[12] http://tds.loctite.com/tds5/docs/3525-EN.PDF

[13] K.E. Carroll, C. Zhang, D. J. Webb, K. Kalli, A. Argyros, M.C.J. Large, "Thermal response of Bragg gratings in PMMA microstructured optical fibers" Optics Express. Vol. 15, No. 14, 8844-8850 (2007).

[14] T. Ishigure, M. Hirai, M. Sato, Y. Koike, "Graded-index plastic optical fiber with high mechanical properties enabling easy network installations. I" J. Appl, Polym. Sci. 91, 404-409 (2004).

[15] E.E. Shafee, "Effect of photodegradation on the [beta]-relaxation in poly(methylmethacrylate)" Polym. Degrad. Stabil. 53, 57-61 (1996). 